\title{
Performance Parameters of Fire Detection Systems
}

Richard Lee Smith

Building and Fire Research Laboratory

Gaithersburg, Maryland 20899

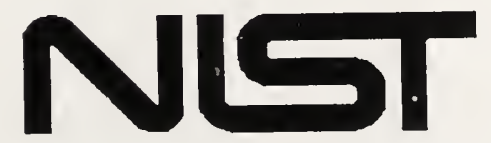

United States Department of Commerce

Technology Administration

$Q C$

National Institute of Standards and Technology

100

.056

N0.5439

1994 

NISTIR 5439

\section{Performance Parameters of Fire Detection Systems}

Richard Lee Smith

June 1994

Building and Fire Research Laboratory

National Institute of Standards and Technology

Gaithersburg, MD 20899

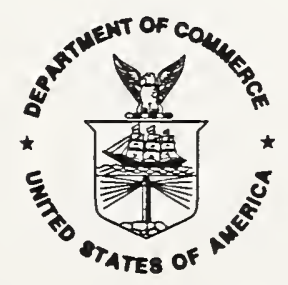

U.S. Department of Commerce

Ronald H. Brown, Secretary

Technology Administration

Mary L. Good, Under Secretary for Technology

National Institute of Standards and Technology

Arati Prabhakar, Director 



\section{Contents}

1 Introduction 1

2 Detection System Requirements 3

2.1 Detector Characterization for Short Times . . . . . . . . . 5

2.2 Detector Characterization for Longer Times . . . . . . . . . . 7

2.3 Value of Information . . . . . . . . . . . . . . 10

3 Conclusions 13 


\begin{abstract}
This report is a formal, functional analysis of fire detection systems' requirements. The performance parameters of fire detection systems are given as conditional probabilities. These parameters are identified by the objective analysis of the functions of a fire detection system. It is demonstrated that using the false alarm rate to specify the malfunctioning of a threshold detection system is inadequate. The principal function of fire detection systems is identified as the notification of anti-fire agents of the probability of an unwanted fire. The evaluation of the information provided by a detector system is centeral to its worth.
\end{abstract}

Keywords: Detection, Fire Detection, Probability, Fire Research 


\section{INTRODUCTION}

The early, highly accurate detection of unwanted fires is very desirable. Normally humans are the best fire detection systems. Humans can use multiple senses and intelligent processing to detect unwanted fires. Whenever people are incapacitated (e.g., asleep) or are not present in the space in which the fire originates, a reliable, automatic fire detection system can be of significant value.

The purpose of this paper is to describe the formal analysis of the desired function of fire detection systems, to identify the performance parameters of fire detection systems, and to demonstrate the approach to evaluating the information provided by such systems. The performance parameters of fire detection systems are expressed as conditional probabilities.

Present conventional detectors are threshold detectors. A typical threshold detector is one that senses a physical parameter and sets off an alarm when the observed parameter exceeds some predetermined value. For example, when the amount of light scattered in a photoelectric smoke detector exceeds some value it sets off a fire alarm. Some threshold detectors have been programmed so that the threshold must be exceeded for some time before an alarm will sound. In addition, some "smart" detectors are multiple parameter, threshold detectors[1][2][3]. For these detectors, the threshold is defined as a volume in a space in which each measured parameter defines a dimension. Whenever the coordinates of the status fall outside this threshold volume the alarm sounds.

For any physical parameter that is used in an effort to detect an unwanted fire, there are sources for this physical parameter other than the unwanted fire. We will refer to this portion of the signal as the background signal, the noise signal, or the environmental signal for that particular physical parameter. In conventional threshold detectors the threshold value is set at a level greater than the maximum expected background signal, plus a safety margin that is a compromise between having too many false alarms and missing the detection of unwanted fires. 
Having a good knowledge of the background signals that a threshold detector will experience is of significant value in optimizing its performance. However, this sort of information is difficult to obtain in a generic form because every structure is unique, and often the background for a particular structure is not constant over a time that correspond to the useful life of the detector. Because of the possibility of wide variations in the parameter being sensed for an arbitrary structure, the threshold levels are frequently set unnecessarily high and therefore do not provide early detection; or they are set too low and therefore produce many false alarms. In the next section we will describe the requirements of a detection system. 


\section{Detection System Requirements}

This section examines the parameters that can be used to specify the performance of a detection system. We will see that it is difficult to separate the detector's performance from the characteristics of its environment including any unwanted fires. We will see that the requirements are driven by the cost of the new information provided by the detection system, the cost of the actions taken, and the resulting savings.

The function of a detection system is to provide new information. In particular, a fire detection system is to inform an anti-fire agent that there is some probability of an unwanted fire. This agent is a person or mechanism that is capable of making decisions or taking action relative to one or more of the following:

1. investigating the presence and attributes of the fire,

2. fighting the fire,

3. escaping, •

4. notifying others, or

5. helping others to escape.

All of these actions should be designed to reduce fire losses. A detector system should provide information that will facilitate the anti-fire agents developing and executing a plan of action that will minimize losses.

The value of the new information provided by the detection system, $V_{d}$, is given by the equation

$$
V_{d}=L-L_{d}-D-R_{d}
$$

where $L$ is the losses without the detection system, $L_{d}$ is the losses with the detection system which includes the losses due to missing a real fire, $D$ is the cost 
of installing and operating the detection system, and $R_{d}$ is the cost of responding to the detection system for all the instances that it calls for action. There is large uncertainty associated with all these quantities. The rational response to a fire detection system should be consistent with the accuracy of information supplied by the detector system and the other information involved in equation 2.1. For example, one has to determine whether a rational response is to just investigate the presence of a fire or whether one should immediately call the fire department. The cost of responding to alarms in dollars, injuries, and lives should be less than the reduction in fire loses.

Early detection requires the detection of small unwanted fires. We are using an unspecified attribute (or attributes) of the fire to determine its size (i.e., smallness or bigness). The sizes we are interested in reflect a measure of the damage an unwanted fire can do. This in turn depends upon the space the detector is monitoring and the contents (including occupants) of this space and the value assigned to the structure and its various contents. The attribute that determines size may be the physical parameter(s) that the detector reacts to, or one closely correlated to the parameter of primary interest. However, this problem of how to determine the size of the fire will be left unresolved since it is very difficult and not critical for our present discussion.

We will classify all fires as belonging to one of three types: none or very small (i.e. smaller than the threshold fire), threshold, or big fires. None or very small fires are below the level we wish to detect. Threshold fires determine the range of fires over which a properly working threshold detector will detect fires. A big fire is any fire larger than a threshold fire. For any threshold fire detection system we have the following possibilities:

1. There is no alarm and there is no unwanted fire;

2. There is no alarm and there is a threshold unwanted fire;

3. There is no alarm and there is a big unwanted fire;

4. The alarm went off and there is no unwanted fire;

5. The alarm went off and there is a threshold unwanted fire; and

6. The alarm went off and there is a big unwanted fire. 
Items $2,3,4$, and 6 can be considered as malfunctions or false alarms. The reporting of malfunctions 2 and 6 is difficult because it is hard for most people to know the size of a fire. In the case of a big unwanted fire it may be difficult to determine whether the alarm operated properly. Therefore, in some cases malfunction 3 can be determined, but in some it cannot. Malfunction 4 is responsible for most reported false alarms and is easy to determine if there is a human present. Because of these considerations, it is difficult to determine that a detector has malfunctioned for all these cases.

\subsection{Detector Characterization for Short Times}

Before we examine these six cases in more detail, we need to introduce the concept of the response time of a detector. In general a detector looks at some physical quantity (such as temperature or the rate of change of temperature) and when that quantity is sufficiently large the detector issues an alarm or a signal. If this sufficiently large change in the physical quantity occurs instantaneously, the time between the change and the time the alarm signal is issued is approximately the response time of the detector $t_{r}$.

For a detector in a particular situation we would like to know the probability for the above six cases. Let us consider the following propositions ${ }^{1}$ for a time interval $t_{r}$ at some time $t$ :

- $f$ : There is no fire or the fire is smaller than the threshold size for a fire.

- $F_{1}$ : The fire is a threshold size fire.

- $F_{2}$ : The fire is larger than the threshold size for a fire.

- A: The alarm goes off during this time interval.

- $a$ : The alarm does not go off during this time interval.

We assume that a detector responds only once to an event that triggers it.

We note that $p(A B \mid K)$ is the probability of propositions $A$ and $B$ being true given $K$; where $K$ is the knowledge we have about the situation, including the

\footnotetext{
${ }^{1}$ A proposition is a statement which is either true or false. A proposition will be represented by a capital letter (e.g., Z) and its negation by the same letter but in lower case (e.g., z).
} 
location of the sensors, the characteristics of the physical space, the contents of the space, the detection system, and the fire. Then we can construct the following contingency table, Table 2.1 [4].

\begin{tabular}{|l|l|l|l|l|}
\hline & $F_{1}$ & $F_{2}$ & $f$ & Sum \\
\hline$A$ & $p\left(A F_{1} \mid K\right)$ & $p\left(A F_{2} \mid K\right)$ & $p(A f \mid K)$ & $p(A \mid K)$ \\
$a$ & $p\left(a F_{1} \mid K\right)$ & $p\left(a F_{2} \mid K\right)$ & $p(a f \mid K)$ & $p(a \mid K)$ \\
Sum & $p\left(F_{1} \mid K\right)$ & $p\left(F_{2} \mid K\right)$ & $p(f \mid K)$ & 1 \\
\hline
\end{tabular}

Table 2.1: Alarm and Fire Contingency Table

In the construction of Table 2.1 we have made use of the relationship

$$
p\left(\alpha F_{1} \mid K\right)+p\left(\alpha F_{2} \mid K\right)+p(\alpha f \mid K)=p(\alpha \mid K)
$$

and

$$
p(A \beta \mid K)+p(a \beta \mid K)=p(\beta \mid K) .
$$

where $\alpha$ is either $A$ or $a$ and $\beta$ is either $F_{1}, F_{2}$, or $f$ since the propositions corresponding to $\alpha$ and $\beta$ form exclusive and exhaustive sets [5]. Also, we note that

$$
p(A \mid K)+p(a \mid K)=1
$$

and

$$
p\left(F_{1} \mid K\right)+p\left(F_{2} \mid K\right)+p(f \mid K)=1
$$

since we are summing over an exhaustive and exclusive set in both cases [5].

The phrase "false alarm rate" is not consistently defined in the literature [6] so it is difficult to relate to our analysis. One meaning in use is that the false alarm rate is the number of times the alarm goes off when there is no fire divided by the number of times there is a fire. If we assume this definition, we would have the false alarm rate equal to $p\left(A f \mid K^{\prime}\right) /\left[p\left(F_{1} \mid K\right)+p\left(F_{2} \mid K\right)\right]$. We see that this expression used only one out of the five parameters needed to characterize a detector and only one out of the four malfunction parameters. Ideally, one would want to have the values for all six probabilities.

In any case, pragmatic consideration requires that the detector's parameters be estimated for times large compared to the response time of the detector. Therefore, we now will develop probabilistic expressions for times large compared to the response time of the detector. 


\subsection{Detector Characterization for Longer Times}

We now want to consider times long compared to the response time of a detector, but still short compared to the expected time for a fire to occur. For having a proper perspective, we note that the response time of a detector should be in the neighborhood of a second while the expected time for a fire to occur in a residence is of the order of a hundred years. We are interested in times of the order of one year for the characterization of detectors.

We note that $p(a f \mid K)$ is approximately one and that all the other probabilities are very small compared to one for a normal residence and a detector that is not obviously defective.

We wish to determine the probability of the alarm going off once when there is no fire in $n$ consecutive time intervals. We will look at the probability of the alarm going off in the one interval, in two intervals, in three intervals, and finally we infer the expression for the alarm going off in $n$ consecutive time intervals.

For one time interval, say the $j^{\text {th }}$ one, the probability of an alarm going off and no fire can be written as $p\left(A t_{r j} f \mid K\right)$ where the proposition ${ }^{2} t_{r j}$ is: The time interval is the $j^{\text {th }}$ time interval of size $t_{r}$.

For two time intervals we evaluate the probability of the alarm going off either in the $j^{\text {th }}$ or $(j+1)^{t h}$ time interval of duration $t_{r}$. This can be written in conditional probability notation as $p\left(A t_{r j} f+A t_{r(j+1)} f \mid K\right)^{3}$. Using the additive rule for probabilities.[4] we may expand this expression and write

$$
p\left(A t_{r j} f+A t_{r(j+1)} f \mid K\right)=p\left(A t_{r j} f \mid K\right)+p\left(A t_{r(j+1)} f \mid K\right)-p\left(A t_{r j} t_{r(j+1)} f \mid K\right)
$$

Since we cannot be in two time intervals at the same time, we see that

$$
p\left(A t_{r j} t_{r(j+1)} f \mid K\right)=0 .
$$

Therefore equation 2.6 becomes

$$
p\left(A t_{r j} f+A t_{r(j+1)} f \mid K\right)=p\left(A t_{r j} f \mid K\right)+p\left(A t_{r(j+1)} f \mid K\right)
$$

\footnotetext{
${ }^{2}$ The conventional usage for propostions would require the use of a capital letter $(T)$ for this propostion. However, since this might be taken to stand for the temperature instead of time, the lower case letter was used.

${ }^{3} p(A+B \mid C)$ means the probability of $A$ or $B$ given $C$.
} 
We can expand both expressions on the right using the fundamental rules for probabilities that state that

$$
p(X Y \mid Z)=p(X \mid Y Z) p(Y \mid Z)
$$

so we have

$$
p\left(A t_{r j} f+A t_{r(j+1)} f \mid K\right)=p\left(A f \mid K t_{r j}\right) p\left(t_{r j} \mid K\right)+p\left(A f \mid K t_{r(j+1)}\right) p\left(t_{r(j+1)} \mid K\right)
$$

We note that

$$
p\left(t_{r(j+1)} \mid K\right)=p\left(t_{r j} \mid K\right)
$$

If we substitute for $p\left(t_{r(j+1)} \mid K\right)$ in equation 2.10 we obtain

$$
p\left(A t_{r j} f+A t_{r(j+1)} f \mid K\right)=p\left(t_{r j} \mid K\right)\left[p\left(A f \mid K t_{r j}\right)+p\left(A f \mid K t_{r(j+1)}\right)\right]
$$

For three time intervals we ask what is the value of $p\left(A t_{r j} f+A t_{r(j+1)} f+A t_{r(j+2)} f \mid K\right)$. If we proceed as we did above we have

$$
\begin{gathered}
p\left(A t_{r j} f+A t_{r(j+1)} f+A t_{r(j+2)} f \mid K\right)=p\left(A t_{r j} f \mid K\right) \\
+p\left(A t_{r(j+1)} f+A t_{r(j+2)} f \mid K\right) \\
-p\left(A t_{r j} f\left[A t_{r(j+1)} f+A t_{r(j+2)} f\right] \mid K\right)
\end{gathered}
$$

Since we cannot be in two time intervals at the same time, we see that

$$
p\left(A t_{r j} f\left[A t_{r(j+1)} f+A t_{r(j+2)} f\right] \mid K\right)=0 .
$$

Therefore equation 2.13 becomes

$$
\begin{gathered}
p\left(A t_{r j} f+A t_{r(j+1)} f+A t_{r(j+2)} f \mid K\right)=p\left(A t_{r j} f \mid K\right) \\
+p\left(A t_{r(j+1)} f+A t_{r(j+2)} f \mid K\right)
\end{gathered}
$$

If we expand the last expression using the additive rule for probabilities we obtain

$$
\begin{gathered}
p\left(A t_{r j} f+A t_{r(j+1)} f+A t_{r(j+2)} f \mid K\right)=p\left(A t_{r j} f \mid K\right)+p\left(A t_{r(j+1)} f \mid K\right) \\
+p\left(A t_{r(j+2)} f \mid K\right)
\end{gathered}
$$

We can expand all the expressions on the right so we have

$$
\begin{gathered}
p\left(A t_{r j} f+A t_{r(j+1)} f+A t_{r(j+2)} f \mid K\right)=p\left(A f \mid K t_{r j}\right) p\left(t_{r j} \mid K\right) \\
+p\left(A f \mid K t_{r(j+1)}\right) p\left(t_{r(j+1)} \mid K\right) \\
+p\left(A f \mid K t_{r(j+2)}\right) p\left(t_{r(j+2)} \mid K\right)
\end{gathered}
$$


Using equation 2.11 we have

$$
\begin{gathered}
p\left(A t_{r j} f+A t_{r(j+1)} f+A t_{r(j+2)} f \mid K\right)=p\left(t_{r j} \mid K\right)\left[p\left(A f \mid K t_{r j}\right)\right. \\
\left.+p\left(A f \mid K t_{r(j+1)}\right)+p\left(A f \mid K t_{r(j+2)}\right)\right]
\end{gathered}
$$

We see that this equation can be expanded readily to $n$ time intervals. If we define $P\left(X \mathcal{T}_{r n} \mid K\right)$ such that

$$
P\left(X \mathcal{T}_{r n} \mid K\right)=p\left(X t_{r j}+X t_{r(j+1)}+\ldots+X t_{r(j+n-1)} \mid K\right)
$$

we have after letting $j=1$ that

$$
P\left(A \mathcal{T}_{\text {rn }} f \mid K\right)=\sum_{k=1}^{n} p\left(A t_{r k} f \mid K\right)
$$

where all the cross terms drop out because we cannot be in two time intervals at once. Proceeding as before we may write

$$
P\left(A \mathcal{T}_{r n} f \mid K\right)=\sum_{k=1}^{n} p\left(A f \mid K t_{r k}\right) p\left(t_{r k} \mid K\right)
$$

If we have

$$
p\left(A f \mid K t_{r i}\right)=p\left(A f \mid K t_{r j}\right)
$$

for all $i$ 's and $j$ 's in the ranges 1 to $n$ and 1 to $l$, we could write

$$
\frac{P\left(A \mathcal{T}_{r n} f \mid K\right)}{P\left(A \mathcal{T}_{r l} f \mid K\right)}=\frac{n}{l}
$$

While one can find many situations for which equation 2.22 is true, there are many situations when it is not true. For example, one would expect equation 2.22 to be valid often for time intervals only a few seconds apart, because at this time scale little changes. However, if the time separating the two terms of equation 2.22 were 12 hours apart one would not be surprised if equation 2.22 was not valid. For example, consider the probability of having a false alarm while preparing dinner verse having a false alarm 8 or 12 hours later in the middle of the night. The above can be repeated for $F_{1}$ and $F_{2}$ in place of $f$. Then these results can be repeated with $a$ substituted for $A$. 
Under the assumption that the false alarm rate is the number of times the alarm goes off when there is no fire divided by the number of times there is a fire, we have the false alarm rate equal to $P\left(A \mathcal{T}_{\text {rn }} f \mid K\right) /\left[P\left(\mathcal{T}_{r n} F_{1} \mid K\right)+P\left(\mathcal{T}_{r n} F_{2} \mid K\right)\right]$.We see that the conventional false alarm rate does not include all the malfunctions a detection system may have. Therefore, using the false alarm rate to specify the malfunctioning of a detection system is inadequate. Of the six probabilities $P\left(A \mathcal{T}_{r n} F_{1} \mid K\right), P\left(A \mathcal{T}_{r n} F_{2} \mid K\right), P\left(A \mathcal{T}_{r n} f \mid K\right), P\left(a \mathcal{T}_{r n} F_{1} \mid K\right), P\left(a \mathcal{T}_{r n} F_{2} \mid K\right)$, and $P\left(a \mathcal{T}_{r n} f \mid K\right)$ only five are independent since the sum of the six is unity. Therefore, the complete specification of the performance of a detection system in a particular situation requires the determination of at least five of these six probabilities. Because $P\left(a \mathcal{T}_{r n} f \mid K\right)$ is approximately one, it is best to use the five other probabilities. Otherwise $P\left(a \mathcal{T}_{r n} f \mid K\right)$ would have to be determined to an accuracy comparable to the smallest of the other five probabilities.

In order to compare different detector systems it is important to realize that $K$ should be the same for each system. We recall that $K$ is what is know to be true. This could including the location of the sensors, the characteristics of the physical space, the contents of the space, the detection system, and the fire. In practice one might want a standardized set of $K$ 's that would facilitate the comparison of detectors. Furthermore, besides having $K$ the same for various detectors, one needs a set of $K$ weighted so they reflect their probability of occurrences in actual usage.

\subsection{Value of Information}

In closing this section we return to consideration of equation 2.1. If the cost of responding to an alarm is $C_{a}$, then $R_{d}$ of equation 2.1 can be written as

$$
R_{d}=C_{a} p(A \mid K) .
$$

Normally $C_{a}$ is not a single value but can be various values with various probabilities. For example the cost of responding to an alarm could include the dollar cost of operating the fire department equipment, the value of injuries to fire department personnel, or the deaths of fire department personnel that occur in responding to the alarm. We can write the probability of $\operatorname{cost} C_{a}$ as $p\left(C_{a} \mid K\right)$. We have assumed that the cost of responding to an alarm is irrelevant to the existence of an alarm 
being sounded. That is we have assumed the following equation is valid

$$
p\left(A C_{a} \mid K\right)=p(A \mid K) p\left(C_{a} \mid K\right) .
$$

The expected cost of responding to an alarm can be written as

$$
E\left(C_{a}\right)=\int_{C_{l}}^{C u} C_{a} p\left(C_{a} \mid K\right) d C_{a}
$$

where $C_{l}$ is the lowest or minimum value for the cost of responding and $C_{u}$ is the upper or maximum value.

We have previously defined $A$ as the alarm sounding. We will define $A(n)$ as the proposition "The alarm $l_{i}$ in structure $s_{j}$ will go off $n$ times in the time interval between and including $t_{k}$ and $t_{m}$ during which time there are no fires." The expected number of alarms in this time interval is

$$
E(n)=\sum_{n=0}^{N} n p(A(n) \mid K)
$$

where $N$ is the maximum value $n$ takes.

When we have repeated, independent trials (Bernoulli trials)[7], we may write

$$
p(A(n) \mid K)=C(N, n)[p(A \mid K)]^{n}[p(a \mid K)]^{N-n}
$$

where

$$
C(N, n)=\frac{N !}{n !(N-n) !} .
$$

Therefore, the expected total cost of responding to all the alarms is

$$
R_{d}=E\left(C_{a}\right) E(n)
$$

We may expand $p(A(n) \mid K)$ so this becomes

$$
R_{d}=E\left(C_{a}\right) \sum_{n=0}^{N}\left[p\left(A(n) F_{1} \mid K\right)+p\left(A(n) F_{2} \mid K\right)+p(A(n) f \mid K)\right] .
$$

As one would expect, the cost of responding to the alarms is a function of the various probabilities leading to an alarm whether there is or is not an unwanted fire. 
The computation of $L_{d}$ is more difficult than that for $R_{d}$. We proceed as before and write for the expected value for $L_{d}$ the following

$$
E\left(L_{d}\right)=\int_{L_{l}}^{L_{u}} L_{d} p\left(L_{d} \mid K\right) d L_{d} .
$$

where $L_{l}$ is the lowest or minimum value for the losses with the detector system and $L_{u}$ is the upper or maximum value. The value of $E\left(L_{d}\right)$ can be greater than $L$. For instance if the installation of a detector system was accompanied by the abandonment of some other more effective fire safety system, the losses could increase.

Let us assume that the addition of the detection system is the only change made to the structure in question. In this case the losses are some function of when the fire is detected relative to its size compared to when the fire would have been detected without the detection system. This seem to be highly situation dependent and we can make no more general comments about it.

We see that the value of adding a detection system depends not only on the performance of the detection system but on the particular situation it is used in. The evaluation of whether it is worth while should be estimated for the particular situation it will be used in. 


\section{Conclusions}

The principal function of a detection system has being identified as providing new information to an anti-fire agent. An expression that shows the value of the information provided by a detector system was developed. The value of information determines what information can economically be collected and therefore, what detection systems are rational to install.

Starting from times comparable to the response time of a detector, we derived the five parameters, using conditional probabilities, that completely characterize a threshold fire detection system.

The following can be considered as malfunctions or false alarms for a fire detection system:

- There is no alarm and there is a threshold unwanted fire;

- There is no alarm and there is a big unwanted fire;

- The alarm went off and there is no unwanted fire; and

- The alarm went off and there is a big unwanted fire.

It is difficult to determine that a detector has malfunctioned for all these cases. A commonly used meaning for the false alarm rate is demonstrated to be inadequate.

Finally, the critical role of $K$ in the analysis of detection systems is made explicit. To compare detector systems $K$ needs to be specified. That is, we needed to identify or control all the factors that will influence the probabilities relating to the detection of fires. 


\section{BIBLIOGRAPHY}

[1] Ellwood, S., Analogue Optical/Heat Fire Detection in International Conference on Automatic Fire Detection, Editor H. Luck, 1989.

[2] Ishii, H., T. Ono, Y. Yamauchi, and S. Ohtani, Algorithm for Improving the Reliability of Detection with Processing of Multiple Sensors Signal, in International Conference on Automatic Fire Detection, Editor H. Luck, 1989.

[3] Okayama, Y., Primitive Study on Fire Detection Method Controlled by Artificial Neural Net, in International Conference on Automatic Fire Detection, Editor H. Luck, 1989.

[4] Tribus, M., Rational Descriptions, Decisions and Designs, Pergamon Press, 1989.

[5] Jeffreys, Harold, Theory of Probability, $3^{\text {rd }}$ Edition, Clärendon Press, Oxford 1983

[6] Dubivsky, P. M., and R. Bukowski, False Alarm Study of Smoke Detectors in Department of Veterans Affairs Medical Centers, NISTIR 89-4077,

[7] Hamming, Richard W., The Art of Probability for Scientists and Engineers, Addison-Wesley Publishing Co.,1991 p55 


\begin{tabular}{|c|c|c|c|}
\hline \multirow{2}{*}{$\begin{array}{l}\text { U.S. DEPARTMENT OF COMMERCE } \\
\text { NATIONAL INSTITUTE OF STANDARDS AND TECHNOLOGY }\end{array}$} & \multicolumn{3}{|c|}{ (ERB USE ONLY) } \\
\hline & \multicolumn{2}{|c|}{ TTROL NUMBER } & DIVISION \\
\hline \multicolumn{4}{|l|}{ 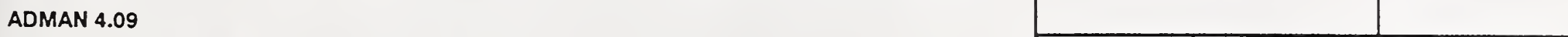 } \\
\hline MANUSCRIPT REVIEW AND APPROVAL & $\begin{array}{l}\text { PUBLCATION REPOR } \\
\text { NISTIR } 5439\end{array}$ & BER & CATEGORY CODE \\
\hline $\begin{array}{l}\text { STRUCTIONS: ATTACH ORIG } \\
\text { IE SECRETARY, APPROPRIATE }\end{array}$ & $\begin{array}{l}\text { PUBLCATION DATE } \\
\text { June } 1994\end{array}$ & & BER PRINTED PAGES \\
\hline
\end{tabular}
TITLE AND SUBTITLE (CITE IN FULL)

Performance Parameters of Fire Detection Systems

\begin{tabular}{|l|l}
\hline CONTRACT OR GRANT NUMBER & TYPE OF REPORT AND/OR PERIOD COVERED
\end{tabular}

\begin{tabular}{|l|l|l|}
\hline AUTHOR(S) (LAST NAME, FIRST INITIAL, SECOND INITIAL) & PERFORMING ORGANIZATION (CHECK (X) ONE BOX) \\
Smith, Richard L. & $\begin{array}{l}\text { NIST/GAITHERSBURG } \\
\text { NIST/BOULDER } \\
\text { JILA/BOULOER }\end{array}$ \\
\hline
\end{tabular}

LABORATORY AND DIVISION NAMES (FIRST NIST AUTHOR ONLY)

Building and Fire Research Laboratory/Fire Science Division.

SPONSORING ORGANIZATION NAME AND COMPLETE ADDRESS (STREET, CITY, STATE, ZIP)

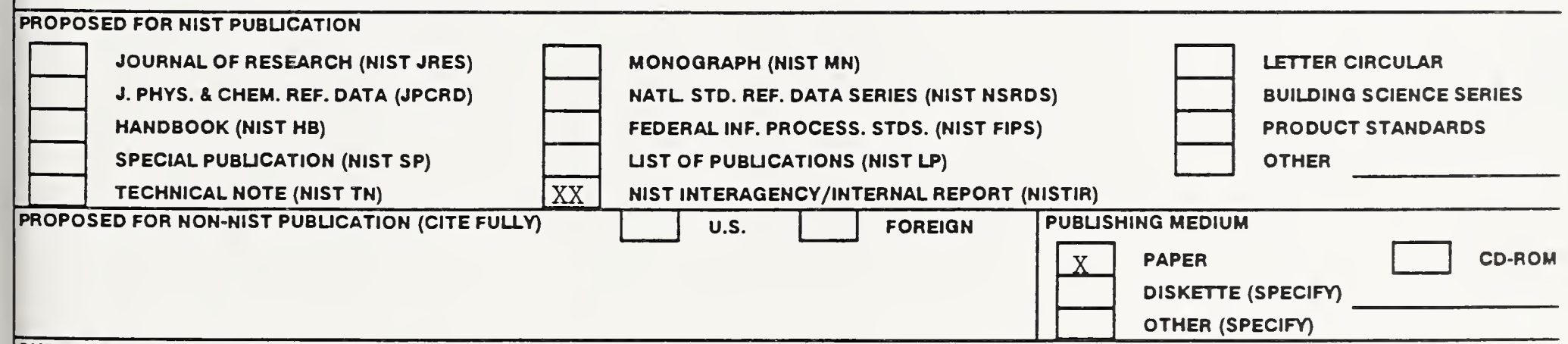

SUPPLEMENTARY NOTES

ABSTRACT (A 2000-CHARACTER OR LESS FACTUAL SUMMARY OF MOST SIGNIFICANT INFORMATION. IF DOCUMENT INCLUDES A SIGNIFICANT BIBLOGRAPHY OR UTERATURE SURVEY, CITE IT HERE. SPELL OUT ACRONYMS ON FIRST REFERENCE.) (CONTINUE ON SEPARATE PAGE, IF NECESSARY.)

This report is a formal, functional analysis of fire detection systems' requirements. The performance parameters of fire detection systems are given as conditional probabilities. These parameters are identified by the objective analysis of the functions of a fire detection system. It is demonstrated that using the false alarm rate to specify the malfunctioning of a threshold detection system is inadequate. The principal function of fire detection systems is identified as the notification of anti-fire agents of the probability of an unwanted fire. The evaluation of the information provided by a detector system is centeral to its worth.

KEY WORDS (MAXIMUM OF 9; 28 CHARACTERS AND SPACES EACH; SEPARATE WITH SEMICOLONS; ALPHABETIC ORDER; CAPITALZE ONLY PROPER NAMES)

detection; fire detection; fire research; probability 

